(Aus dem Laboratorium weiland des Prof. von Basch, Wien.)

\title{
Ueber die Bewegungs- und Hemmungsnerven des Uterus.
}

\author{
Von \\ Dr. Leopold Fellner, kais. Rath, \\ Franzensbad. \\ (Mit 20 Curren im Text.)
}

Im Jahre 1887 habe ich im Laboratorium des Prof. von Basch Studien über die Innervation des Uterus unter Anwendung von Methoden, durch welche die Contractionen des Uterus beim Hunde graphisch dargestellt wurden, vorgenommen. Hierbei habe ich die Wirkungsweise jener Nerven auf den Uterus geprüft, welche nach den Untersuchungen von v. Basch und Hoffmann (1) Bewegungserscheinungen in der Cervix uteri hervorrufen. Ess sind dies die Nn. erigentes, $d . h$. die beiden aus dem Sacralplexus entspringenden und in den Plexus hypogastricus sich einsenkenden Nerven, und die Nn. hypogastrici, d. i. ein Nervenpaar, das aus dem Ganglion mesentericum posterius stammt und sich gleichfalls wie die Nn. erigentes in den Plexus hypogastricus einsenkt.

Eine vorläufige Mittheilung über die Methoden dieser Untersuchungen und ihre Resultate sind im Centralblatt für die medicinischen Wissenschaften im Jahre 1887 erschienen (2). Die ausführliche Mittheilung ist bisher aus äusseren Gründen unterblieben. Leider ist Prof. von Basch vor einem Jahre gestorben, ish werde mich aber strenge an die Beschreibung der Methoden und die Formulirung der Ergebnisse, wie wir sie seiner Zeit zusammengestellt, halten, die wichtigsten Einzelheiten der Untersuchungen mittheilen; und die Curven bringen, welche die Ergebnisse illustriren sollen, die praktischen Schlussfolgerungen aber selbstständig ziehen und begründen. 
Ich fühle mich zu dieser verspäteten Mittheilung um so mehr gedrängt, als ich in allen bis auf die jüngste Zeit auf dem Gebiete der Physiologie des Uterus publicirten Untersuchungen ein näheres Eingehen auf die Wirkungsweise dieser Nerven auf den Uterus vermisse, und meine Ergebnisse sehr wichtige Thatsachen für die Physiologie der Gebärmutter aufdecken, welche nicht nur in theoretischer, sondern auch in praktisch-klinischer Beziehung von grosser Bedeutung sind, da sich aus denselben der Einfluss dieser Nerven auf eine Reihe physiologischer Vorgänge, wie Geburtsverlauf, Conception etc. ergeben wird.

Auch die Methoden, welche hierbei zur Anwendung kamen, sind so ausgebildet, wie solche bisher kein Forscher in der Physiologie der Gebärmutter in Anwendung brachte.

Erwähnt ja auch Kurdinowski (3) in seinen höchst interessanten Untersuchungen über die Physiologie der Gebärmutter, dass bisher keine Methode bekannt wurde, nach welcher vorwurfsfreie Ergebnisse beim Studium auf diesem Gebiete erlangt werden konnten.

Die Angaben der früheren Autoren über die physiologische Wirkung der in Rede stehenden Nerven stimmen bekanntlich nicht überein. Spiegelberg (4) spricht den Nn. hypogastr. und Frankenhäuser (5) den Sacralnerven jede motorische Bedeutung ab. Kehrer (6) und Körner (7) halten beide für motorische Nerven des Uterus. Erst die Untersuchungen von v. Basch und Hoffmann (1) haben mehr Aufklärung, wenigstens bezüglich der motorischen. Wirkung dieser Nerven, gebracht. Sie sahen bekanntlich am Hundeuterus bei aufgeschlitzter Vagina, dass mit Reizung der Nn. hypogastr. die Cervix hinabrückt und der äussere Muttermund sich öffnet. Dem Herabrücken der Cervix geht stets eine Einschnürung am Halse der Cervix in der Gegend des inneren Muttermundes voraus. Sie erklärten diesen Vorgang dahin, dass der active motorische Effect der Nn. hypogastr. in der Contraction der Ringmuskelfasern des Uterus bestehe, während die Locomotion der Cervix und das Oeffnen des Muttermundes passive Vorgänge wären.

Nach Reizung der Nn. erigentes sahen sie in den meisten Fällen eine deutliche Verkürzung des Uteruskörpers und hiermit einhergehend eine Einziehung der Cervix und Schliessung des äusseren Muttermundes. Der primäre active Effect ist hier nach Ansicht dieser Autoren die Contraction der Längsmuskeln, während die anderen Erscheinungen passive Vorgänge wären. 
Durch meine Studien, welche ich im Jahre 1883 im Laboratorium des Prof. v. Basch über die Innervation des Rectums mittelst graphischer Methoden vorgenommen und im Jahre 1894 in demselben Laboratorium ergänzt habe, wurde die Wirkungsweise dieser Nerven sowohl auf die Längsmusculatur, wie die Ringmusculatur des Rectums vollständig klargelegt und wurden dieselben uns zum Wegweiser auch für das Studium der Innervation des Uterus.

Die Ergebnisse dieser Untersuchungen, deren erste jch im Jahre 1884 (8) und deren letzte ich im Jahre 1894 (9) publicirte, waren folgende:

1. Die Reizung der Nn. erigentes bewirkt eine Contraction der Längsmusculatur und eine Erschlaffung der Ringmuskelfasern des Rectums.

2. Die Reizung der Nn. hypogastr. bewirkt einerseits eine Verkürzung der Ringmuskelfasern und andererseits eine Erschlaffung der Längsmuskelfasern des Rectums.

Hierdurch wurde das wichtige Gesetz von dem gekreuzten Verlaufe der Bewegungs- und Hemmungsnerven des Rectums aufgedeckt, welches geeignet ist, einen tiefen Blick in den Mechanismus der Peristaltik des Rectums zu gewähren. „Diese bestünde, wenn man zugleich annimmt, dass das Herabgleiten der physiologischen Reize längs der beiden Nervenbahnen alternirend und in rhythmischer Folge von statten geht, in einer abwechselnden Contraction und Erschlaffung beider Fasersysteme und zwar so, dass die Contraction und Erschlaffung wohl gleichzeitig, aber in antagonistischem Sinne zu Stande kommen."

Dieser in die Innervation des Rectums gewonnene Einblick musste uns schon von vornherein die Vermuthung nahe legen, dass auch beim Uterus ähnliche Innervationsgesetze aufgedeckt werden könnten, da er ja von denselben Nerven innervirt wird, doch war mit Rücksicht auf den complicirten anatomischen Bau dieses Organs und die Verschiedenheit zwischen Cervix und Corpus uteri in morphologischer und functioneller Beziehung, gegenüber der einfachen gleichmässigen Anlage des Rectums, nicht ausgeschlossen, dass hier auch die Innervationsverhältnisse andere sein könnten.

Der Gang unserer Untersuchungen war folgender: Wir prüften vorerst den Einfluss der Nn. erigentes und der hypogastr. auf die Musculatur der Cervix und nachher auf die Musculatur des Uterus. Unsere Versuche wurden an curaresirten, künstlich geathmeten 
Hunden ausgeführt, und stimmen unsere Erfahrungen in Bezug auf die Reizbarkeit des Uterus gegenüber dem elektrischen Strome durchaus nicht mit den Mittheilungen, welche Kurdinowski (3) u. A. gemacht, die den elektrischen Reizen eine geringe Einwirkung auf den Uterus beimessen, denn wir haben den faradischen Strom sowohl bei jungfräulichem Uterus, wie bei trächtigen Thieren und solchen, welche schon geworfen hatten, mit sehr geringen Ausnahmen wirksam gefunden. Es scheint demnach, dass der elektrische Strom viel wirksamer ist, wenn er auf den Wegen der Nervenbahnen zu einem Organ geleitet wird, als wenn er direct auf die Muskelfaser des Organs einwirkt.

Es kamen vier verschiedene Methoden zur Anwendung, auf deren ausführliche Beschreibung ich hier nicht eingehen kann, ich will mich darauf beschränken, das anzuführen, was zur richtigen Deutung der Curven nöthig ist.

A. Mittelst einer myographischen Zange gleicher Construction, wie sie Oser (10) bei seinen Untersuchungen über die Pylorusbewegungen anwendete, wurden die Contractionen und Erschlaffungen der Cervix uteri graphisch dargestellt.

Es ist das eine federnde Zange, deren innere Branchen bei jeder Cervixcontraction aneinander gedrängt werden und durch die correspondirende Bewegung der äusseren Branchen eine in der einen Branche befindliche Schraube an eine mit Wasser gefüllte Kapsel angedrückt wird, welche mit dem $v$ on B asch'schen Wellenschreiber in Verbindung gebracht wird. Die Cervix wurde mittelst Fäden an die Zange befestigt, damit letztere nicht herausgleite.

\section{Versuch der Hypogastricusreizung bei gleichzeitiger Athmungssuspension.}

In Folge der Dyspnoe nach Athmungssuspension entstehen Contractionen, welche ähnlich den spontanen Contractionen sind. Inmitten derselben wird der $\mathrm{N}$. hypogastr. gereizt, die Curve sinkt $a b$, der Hebel zeichnet eine lange gerade Linie [Fig. $2 a$ und $b]^{1}$ ).

Diese Linie, welche die Ruhepause bis zu den nächsten spontanen Contractionen $c, d$, e bezw. $f$ darstellt, ist nicht nur länger als die Senkungen zwischen den einzelnen spontanen Contractionen, sondern sie unterscheidet sich auch von diesen dadurch, dass die secundären Wellen, welche in den Senkungen nach den spontanen

1) Alle Curven sind von rechts nach links zu lesen. 
Fellner, Die Bewegungs- und Hemmungsnerven des Uteras:

241
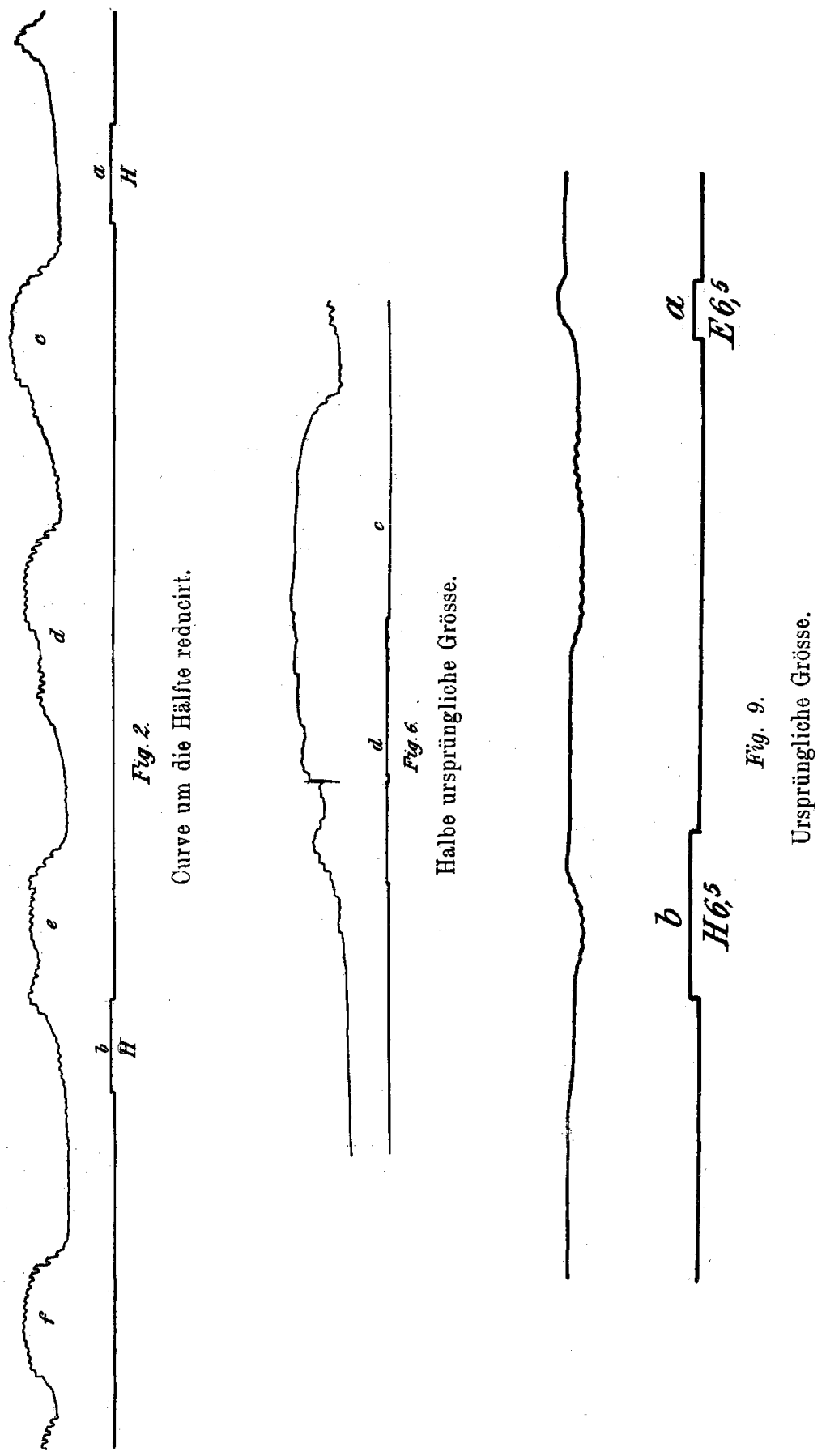
Contractionen sichtbar sind, abnehmen, d. h. dass vollständige Erschlaffung eintritt.

Wie sind nun diese Vorgänge zu erklären? Da die durch Dyspnoe hervorgerufenen Contractionen in der vorliegenden Versuchsanordnung der Ausdruck der Ringmuskelcontractionen der Cervix, d. h. der Verengerung des Cervixlumens sind, und diese Contractionen durch Hypogastricusreizung unterdrückt werden, so müssen im N. hypogastr. Hemmungsfasern für die Ringmusculatur der Cervix sein, welche den Tonus derselben herabsetzten, d. h. eine Erschlaffung derselben bewirken.

\section{Nicotinversuch.}

Es wurde nun eine Pravazspritze einer Lösung von 3 Tropfen Nicotin auf $15 \mathrm{~g}$ Wasser dem Thiere subcutan injicirt. Es trat eine mächtige tetanische Contraction ein. (Fig. 6, Curve c.)

Diese Contraction wurde durch die Hypogastricusreizung d gehemmt, es trat Senkung und vollständige Erschlaffung ein.

\section{Wirkung der Nn. erigentes und hypogastrici auf die Ring- musculatur der Cervix nach elektrischer Reizung. (Faradischer Strom.)}

Es wurden dann eine Reihe von Versuchen angestellt, in welchen die Zange in die Cervix eingeführt wurde und sowohl die Nn. hypogastr. wie die erigentes mittelst je eines Schlittenapparates gereizt wurden, um ihre Wirkung auf die Ringmusculatur zu prüfen.

\section{a) Erigensreizung.}

Nach Reizung der Erigentes tritt eine Contraction ein, welcher eine geringe Senkung vorausgeht und eine längere Senkung unter Secundärwellen nachfolgt (Fig. 9 a, R. a. 6,5).

b) Hypogastricusreizung.

Nach Reizung der Hypogastrici tritt eine Senkung ein. Derselben geht eine kleine Erhebung voraus und ebenso folgt ihr eine (Fig. 9 b, R. a. 6,5).

Mit Zunahme der Stromstärke und Reizungsdauer nimmt der Wellenberg nach Erigensreizung an Höhe zu, aber auch die Senkung ist stärker ausgeprägt.

Auch nach Hypogastricusreizung nimmt die Vertiefung, d. i. die Senkung mit der Stromstärke zu. 
Ergebniss: Bei Erigensreizung tritt daher eine Contraction der Ringmusculatur der Cervix ein. Die primäre Wirkung der Nn. erigentes ist also eine motorische auf die Ringmusculatur. Nach dieser Contraction der Ringmusculatur nehmen wir eine langdauernde tiefe Erschlaffung wahr, welche dieser Contraction folgt und in welcher Secundärwellen sichtbar sind. Diese tiefe Erschlaffung ist nicht blos der Ausdruck des Nachlasses der Ringmuskelcontraction d. i. der Herstellung ihres früheren Tonus, sonst müsste die Curvenlinie zu ihrem Ausgangspunkte zurückkehren. Der absteigende Schenkel der Curve sinkt aber unter die frühere Abscisse, es muss sich demnach auch um die Erschlaffung der anderen Muskelfaserschichte, nämlich der Längsfasern der Cervix handeln, welche erst jetzt, nach dem Aufhören der motorischen Wirkung der Erigensreizung auf die Ringmusculatur, in die Erscheinung tritt.

Wir haben es daher bei der Erigensreizung mit zwei Wirkungen auf die Musculatur der Cervix zu thun: Die eine besteht in der motorischen Wirkung auf die Ringmusculatur, die andere in der erschlaffenden bezw. den Tonus herabsetzenden Wirkung auf die Längsmusculatur der Cervix.

Nach Hypogastricusreizung kommt es zu einer Senkung der Curve, ihre Convexität ist gegen die Abscisse gerichtet. Diese Senkung der Curve kommt dadurch zu Stande, dass die Ringmusculatur erschlafft und die Zangenbranchen, ihrer Elasticität folgend, sich erweitern, wie wir dies ja schon auch in den Versuchen mit Dyspnoe und Nicotininjection wahrgenommen.

Die Nn. hypogastr. wirken demnach erschlaffend auf die Ringmuskeln der Cervix, sie sind Hemmungsnerven für die Ringmusculatur der Cervix.

Bei unseren Untersuchungen über die Innervation des Rectums haben wir gefunden, dass die Nn. hypogastr. auch motorische Fasern führen, welche aber dort die Ringmusculatur motorisch beeinflussen. Per analogiam müssten wir erwarten, dass an der Cervix diese motorische Wirkung an der Längsmusculatur sich zeigt, da doch hier im Gegensatze zum Rectum die hemmende Wirkung der Hypogastr. die Ringmusculatur betrifft.

Die der Senkung vorangehende und nachfolgende geringe Erhebung dürfte wohl auf Contraction der Längsmuskeln der Cervix zurückzuführen sein. Dieser Ausschlag der motorischen Wirkung 
244 Fell ner, Die Bewegungs- und Hemmungsnerven des Uterus.

der Hypogastrici ist aber so gering in diesen Versuchen, welche an einem jungfräulichen Thiere vorgenommen wurden, dass wir nicht berechtigt sind, aus diesen Erscheinungen allein Schlüsse zu ziehen. Aus den nachfolgenden Versuchen wird die motorische Wirkung der Nn. hypogastr. klarer hervortreten.

Es wurden nun Versuche an einem Thiere angestellt, dessen Uterus kräftiger entwickelt war. Da trat:

a) nach Reizung der Erigentes vorerst eine kurzdauernde tiefe Senkung, dann eine langdauernde hohe Contraction, hierauf wieder eine tiefe Senkung unter den Ausgangspunkt ein. Fig. 18 b. Der Wellenberg ist der Ausdruck der Contraction der Ringmusculatur, die Wellenthäler der Ausdruck der hemmenden Wirkung auf die Längsmusculatur der Cervix.

b) Nach Hypogastricusreizung kam es zu einer tiefen Senkung; dieser ging eine Contraction voraus und ebenso folgte ihr eine Contraction (s. Fig. 18 a). Die primäre Wirkung ist die hemmende auf die Ringmusculatur, die secundäre ist die motorische Wirkung auf die Längsmusculatur der Cervix.
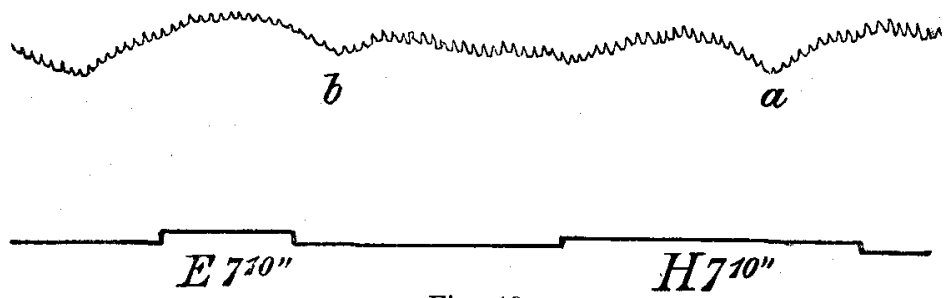

Fig. 18.

Ursprüngliche Grösse.

Die eben angeführten Versuche bestätigen unsere aus den früheren Versuchen gemachten Schlüsse bezüglich der motorischen und hemmenden Fasern der Erigentes und der hemmenden Fasern der Hypogastrici. Aus denselben geht aber auch die motorische Wirkung der Hypogastrici auf die Längsmusculatur der Cervix schon klarer hervor, dieselbe giebt sich in der der tiefen Senkung vorangehenden und nachfolgenden Erböhung der Curve kund.

In dem nun folgenden Versuche hatten wir es mit einem hochträchtigen Thiere zu thun. Der Reizungseffect fiel daher grösser aus.

Es wurde in diesem Versuche die Anordnung derart abgeändert, dass die Zange tiefer eingeführt wurde, da trat nach 
Erigensreizung die Wirkung der Ringmuskeln der Cervix und nach Hypogastricusreizung die Wirkung der Ringmusculatur des Corpus uteri und zwar in seinen untersten Schichten zutage.

a) Nach Erigensreizung tritt eine sehr hohe langdauernde Contraction mit nachfolgender Senkung unter den Ausgangspunkt ein (Fig. 22 a R. a. 710 Sek.).

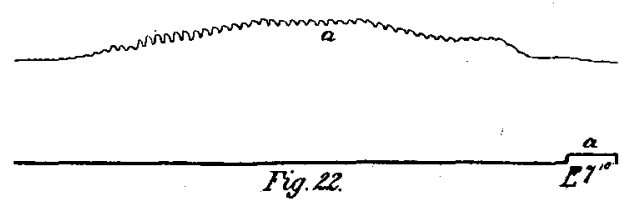

Curve um die Hälfte reducirt.

b) Nach Hypogastricusreizung tritt ebenfalls eine sehr hohe langdauernde Contraction ein, welcher eine längere Senkung vorangeht und nachfolgt (Fig. 23 R. a. 715 Sek.).

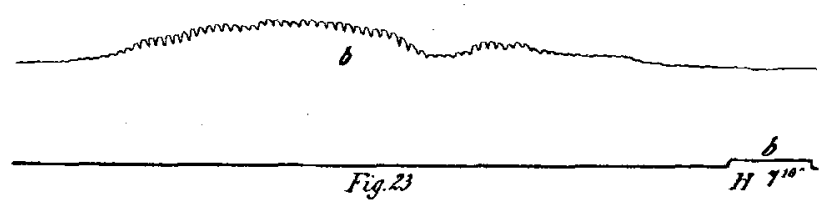

Halbe ursprüngliche Grösse.

Die Analyse dieser Versuche zeigt deutlich, dass der Erigens die Ringmusculatur der Cervix zur Contraction bringt, was wir ja auch schon früher gesehen. Sie zeigt aber in der Curve $b$ in überzeugendster Weise, dass der Hypogastricus der motorische Nerv für die Ringmusculatur des Corpus ateri ist, da seine Reizang die Ringmusculatur dieses Uterusabschnittes zur Contraction bringt.

Aber auch die hemmende Wirkung der Hypogastrici auf die Ringmusculatur der Cervix zeigt sich in der Senkung vor und nach dem Wellenberg und kann bei dieser Versuchsanordnung noch deutlicherdemonstrirt werden. Lassen wir nämlich die Hypogastricusreizung mitten in die Wirkung der

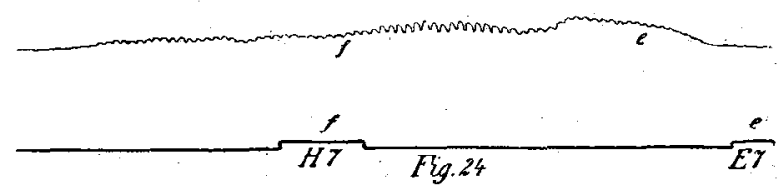

Halbe ursprüngliche Grösse. 
Erigenzreizung einfallen, so wird die Contraction gehemmt, das können wir in Fig. $24 \mathrm{ef}$ und in Fig. $25 \mathrm{~g}$ h wahrnehmen, und zwar tritt die hemmende Wirkung der Hypogastrici um so früher ein, je grösser die Stromstärke. In Fig. 24 war (R. a. 7), da kam es allmälig zur Lösung, in Fig. 25 bei R. a. 6 trat die Hemmung sofort ein.

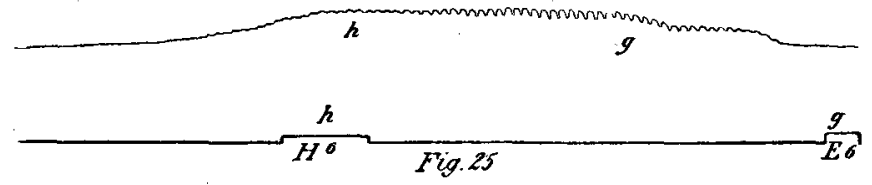

Curve um die Hälfte reducirt.

Die bohen Contractionen nach den vorausgehenden Reizungen waren wahrscheinlich dadurch bedingt, dass bei dem Thiere Wehen eingetreten waren, denn es hatte nach dem Versuche geworfen. Es traten auch spontane Contractionen zwischen den einzelnen Reizungen auf, und man sieht auch den Einfluss derselben in Fig. $23 \mathrm{~b}$ und 24 e, die Curven steigen nicht gleichmässig auf, sondern es tritt mitten im Wellenberg ein Wellenthal ein und dann setzt sich die Contraction fort.

Wir haben aber aus diesen Versuchen in seinem weiteren Verlaufe noch eine interessante und in Bezug auf den Geburtsverlauf wichtige Wahrnehmung gemacht. Es kam nämlich allmälig zur Abnahme der Contractionsstärke in der Ringmusculatur der Cervix, was sich sowohl in der Abnahme der Ordinaten wie der der Abscissen in den Curven kundgab. Die Contractionen in der Ringmusculatur der Cervix wurden fast verschwindend klein, es traten bloss frustrane Contractionen auf. Dies demonstrirt Fig. $27 \mathrm{n} 0$.

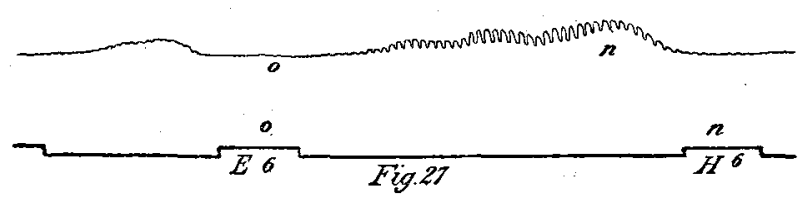

Halbe ursprüngliche Grösse.

Weist das nicht auf die Vorgünge bei der Eröffnungsperiode hin? Ich werde später auf diese Versuche bei meinen theoretischen Schlussfolgerungen zurückkommen. Hier will ich nur hervorheben, dass diese Versuche den unwiderlegbaren Beweis erbringen, dass die $\mathrm{N}$. erigentes die motorischen Fascrn für die Ringmusculatur der Cervix führen and dass die Nn. bypo- 
Fellner, Die Bewegungs- und Hemmungsnerven des Uterus.

gastrici die motorischen Fasern für die Ringmusculatur des Corpus uteri und die Hemmungsfasern für die Ringmusculatur der Cervix enthalten.

Aus den Ergebnissen der bisherigen Versuche können wir daher folgende Schlüsse ziehen:

1. Die Nn. hypogastr. sind die motorischen Nerven für die Längsmusculatur und die hemmenden Nerven für die Ringmusculatur der Cervix. Sie sind aber auch die motorischen Nerven für die Ringmusculatur des Corpus uteri.

2. Die Nn. erigentes sind die motorischen Nerven für die Ringmusculatur und die hemmenden für die Längsmusculatur der Cervix.

Das Gesetz der gekreuzten Innervation, welches wir für das Rectum aufgedeckt haben, gilt daher auch für die Cervix uteri.

\section{B) Belastung der Längsmusculatur des Uterus.}

Wie in unseren Versuchen über die Innervation des Rectums wurde hier der Uteruskörper mit einem belasteten Hebel verbunden. Die Fixirung geschah derart, dass nur die Verkürzung bezw. Verlängerung des seiner Länge nach gespannten Uteruskörpers, aber nicht zugleich die der Hörner und der Vagina, zum Ausdruck gelangen konnte.

Nachstehende Versuche wurden an einer Hündin vorgenommen, welche vor einigen Tagen geworfen hatte, daher der Uterus gross und dick war.

a) Erigenswirkung.

Nach Reizung des Erigens tritt eine Contraction ein, auf welche nach geringer Senkung sich noch eine höhere Contraction aufbaut, dann folgt eine tiefe Senkung. Fig. 28 c. Die Contrac-

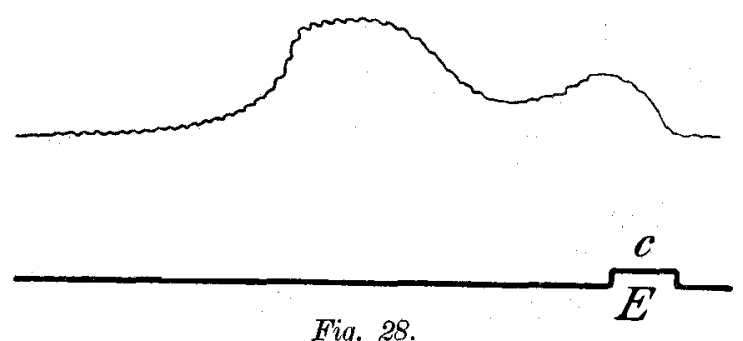

Ursprüngliche Grösse. 
tion betrifft hier die Längsmusculatur des Corpus uteri, während die Senkung unter den Ausgangspunkt nicht nur den Nachlass der Contraction der Längsmusculatur, sondern auch die Erschlaffung der Ringmusculatur andeutet.

b) Hypogastricusreizung.

Die motorische Wirkung der Hypogastr. auf die Ringmusculatur des Corpus uteri haben wir schon in einem früheren Versuche, S. 245 Fig. 23, nachgewiesen.

Die hemmende Wirkung der Hypogastrici auf die'Längsmusculatur des Corpus uteri zeigt sich sehr deutlich in dem nachfolgenden Versuche, welchen Fig. $30 \mathrm{n}$ o demonstrirt.

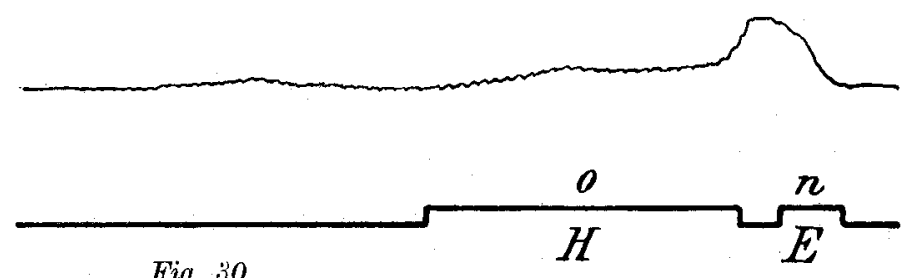

Fig. 30

Ursprüngliche Grösse.

Fällt nämlich die Hypogastricusreizung mitten in die Wirkung des Erigens ein, so wird die Weiterentwicklung der Contraction mehr oder weniger gehemmt. Ist das Intervall zwischen beiden Reizungen ein sehr kurzes, wird die Contraction derart gehemmt, dass nur die kleinere Welle zum Ausdrucke gelangt, und die grössere vollständig unterdrückt wird, so entsteht eine Abortivcontraction, nach welcher es zu langdauernder Erschlaffung kommt, in deren Mitte eine tiefe Senkung erscheint. Diese Vertiefung zeigt weiterhin die hemmende Wirkung der Hypogastrici auf die Längsfasern des Corpus uteri, da sie während der Erschlaffung der Längsmusculatur des Corpus uteri eintritt.

Die Reizung der Nn. erigentes bringt demnach die Längsmuskeln des Uterus zur Contraction und die Ringmusculatur zur Erschlaffung. Der Erigens enthält daher die motorischen Fasern für die Längsmusculatur und die hemmenden für die Ringmusculatur des Corpus uteri.

Die Reizung der Nn. hypogastrici bewirkt eine Contraction der Ringmusculatur und eine Erschlaffung der Längsmusculatur des Corpus uteri.

Es war nun von Interesse zu sehen, wie sich denn die Wirkung 
dieser Nerven bei gleichzeitiger Spannung des Uterus seiner Länge nach, und Application der Zange in die Cervix, kundgiebt.

In diesen Versuchen wurde der Effect auf die Längsmusculatur des Uterus oben geschrieben, während der Effect auf die Ringmusculatur der Cervix in der unteren Curve verzeichnet wurde.

a) Reizung der Erigentes Fig. 35 a.
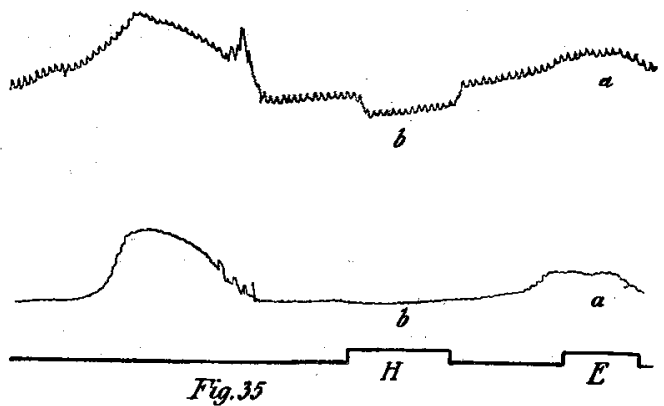

Halbe ursprüngliche Grösse.

In der oberen Curvenlinie tritt vorerst eine kleine Senkung ein, dann eine Contraction, hierauf tiefe Senkung unter den Ausgangspunkt. In der unteren Curve kommt es auch vorerst zur Senkung, dann zu einer ziemlich hohen Contraction und schliesslich zur Senkung.

Curve b: Reizung der Hypogastrici. In der oberen Curvenlinie kommt es zuerst zur tiefen Senkung, dann zu einer langdauernden hohen Contraction und schliesslich zu einer tiefen Senkung unter den Ausgang.

In der unteren Curvenlinie kommt es vorerst zu einer seichten Vertiefung, welche solange dauert wie die Reizung, dann folgt eine hohe, ziemlich lang dauernde Contraction und schliesslich Ersehlaffung.

Bei dieser Anwendung konnte man demnach die Wirkung beider Nerven auf beide Muskelfasersysteme, sowohl des Corpus uteri wie auch der Cervix studiren.

Die Analyse der oberen Curve ergiebt einerseits, dass bej Reizung der Nn. erigentes neben der positiv motorischen Wirkung auf die Längsmusculatur des Uterus, auch eine negativ motorische, d. h. eine erschlaffende Wirkung zutage tritt, und diese betrifft die Ringmusculatur des Uterus. Diese erschlaffende Wirkung manifestirt sich in dem absteigenden Schenkel der Curve, wolcher sich lange und tief unter den Ausgang senkt. 
Die Nn. irigentes führen demnach auch Hemmungsfasern für die Ringmuskeln des Uterus.

Die Analyse der oberen Curve zeigt andererseits auch, dass nach Hypogastricusreizung neben der hemmenden Wirkung derselben auf die Längsmusculatur des Uterus, welche hier so schön in die Erscheinung tritt, auch eine motorische Wirkung zutage tritt, als deren Ausdruck eine hohe Contractionswelle erscheint. Diese motorische Wirkung betrifft hier die Ringmuskeln des Uterus.

Wir können daher aus diesen Untersuchungen über die Innervation des Uteruskörpers folgende Schlüsse ziehen.

1. Die Nn. erigentes sind die motorischen Nerven für die Längsmusculatur und die hemmenden für die Ringmusculatur des Uterus.

2. Die Nn. hypogastrici sind die motorischen Nerven für die Ringmusculatur und die hemmenden für die Längsmusculatur des Uterus.

Um dieses gegenseitige Verhältniss bildlich darzustellen, möchte ich auf die Gestaltsveränderung der sogen. "Faullenzerscheere" hinweisen. Werden die Handgriffe zusammengepresst, wird die Scheere lang und schmal. Werden die Handgriffe auseinandergedrängt, wird die Scheere kürzer und breiter.

Die Analyse der unteren Curvenlinie zeigt ausser der bereits bekannten motorischen und hemmenden Wirkung der Nn. erigentes ganz besonders deutlich die motorische Wirkung der Hypogastrici auf die Längsmusculatur der Cervix, welche hier in einer hohen Contraction sich kundgiebt.

Fällt die Reizung der Hypogastrici inmitten des Effectes der Erigensreizung, so hemmt erstere die Wirkung der letzteren mehr oder weniger, wie dies in Fig. $36 \mathrm{c} d$ ersichtlich wird.

Hier zeigt sich die Wirkung in der Weise abgeändert, dass die Contraction nach Erigensreizung in beiden Uterusschnitten nicht so hoch wie sonst, und die Senkung nicht so tief wie sonst ausfällt, sondern die eine wie die andere eine Resultante beider Reizungen darstellt, und sowohl im Uterus wie in der Cervix rhythmische Contractionen und Erschlaffungen, jedoch auf einer höheren Abscisse, eintreten. Nachträglich kommt es in beiden Curvenlinien zu einer langdauernden Senkung, welche in der Längsmusculatur tiefer ausfällt. 
Fellner, Die Bewegungs- and Hemmungsnerven des Uterus. $\quad 251$

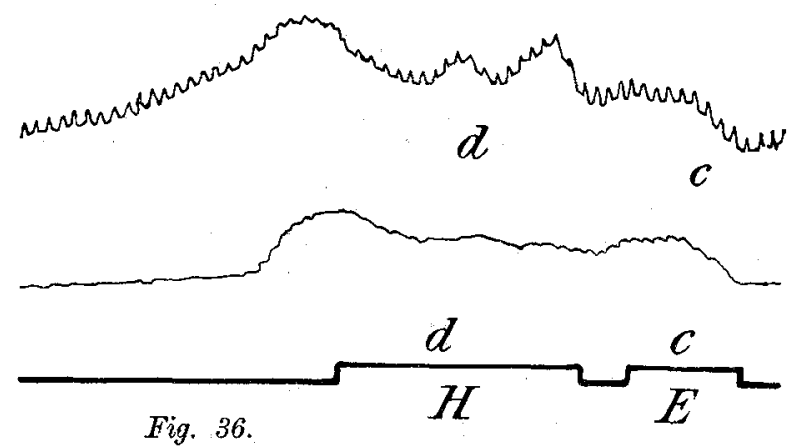

Ursprüngliche Grösse.

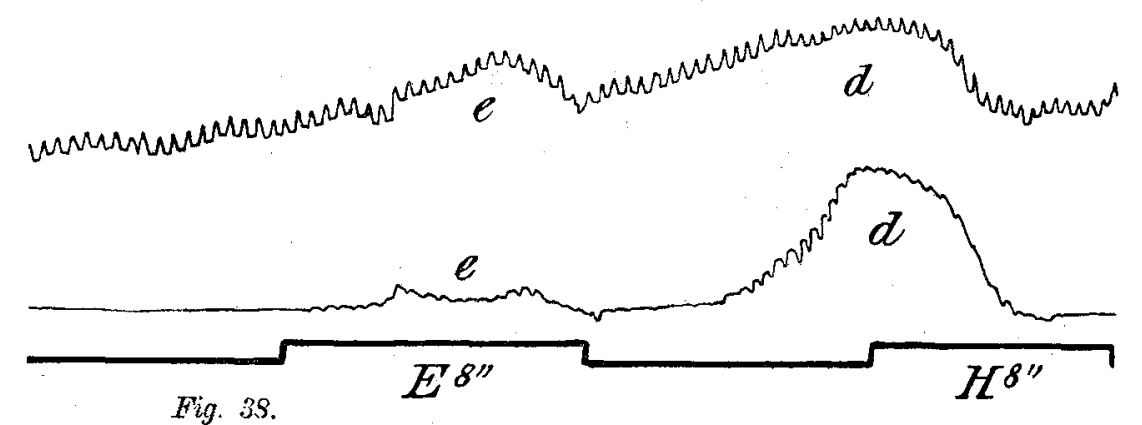

Ursprüngliche Grösse.
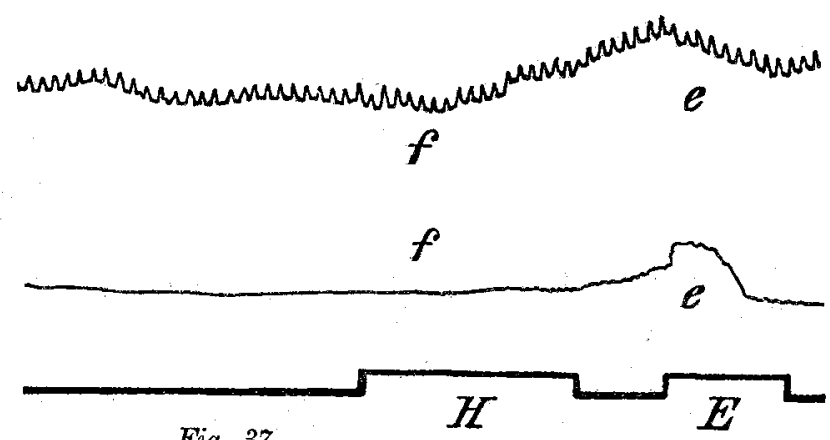

Fig. 37.

Ursprüngliche Grösse. 
Die hemmende Wirkung der Hypogastricusreizung zeigt sich auch deutlich, wenn sie einer Erigensreizung vorausgeht. Siehe Fig. $38 \mathrm{~d}$ e, wo es in beiden Curvenlinien nur zu einer Abortivcontraction kommt, auf welche eine langdauernde Erschlaffung sowohl im Uterus wie auch in der Cervix eintritt.

Fällt die Hypogastricusreizung in eine Erigensreizung hinein, welcher kurz vorher auch eine Hypogastricusreizung voranging, wie in Fig. 37 e f, dann wird die Contraction sowohl der Längsmusculatur des Uterus sowie der Ringmusculatur der Cervix in so hohem Grade gehemmt, dass es nur zu einer geringen Contraction kommt, und eine lange tiefe Erschlaffung eintritt.

Aus diesen Versuchen geht demnach die hemmende Wirkung der Hypogastricusreizung sowohl auf die Längsmusculatur des Corpus uteri wie auf die Ringmusculatur der Cervix auf das Eclatanteste hervor.

Aus allen bisher angeführten Versuchen können wir folgende Schlüsse ziehen:

1. Die Nn. erigentes sind einerseits motorische Nerven für die Längsmusculatur des Uterus und die Ringmusculatur der Cervix, anderseits Hemmungsnerven für die Ringmusculatur des Uterus und für die Längsmusculatur der Cervix.

2. Die Nn. hypogastrici sind einerseits motorische Nerven für die Ringmusculatur des Corpus und die Längsmusculatur der Cervix, andererseits Hemmungsnerven für die Längsmuculatur des Uterus und die Ringmusculatur der Cervix. Es kommt da wie dort das Gesetz der gekreuzten Innervation, nur in umgekehrter Weise zur Anschaung.

Dieses eigenthümliche entgegengesetzte Innervationsverhältniss zwischen Corpus uteri und Cervix ist von vornherein eigentlich sehr auffallend und schwer verständlich. Man sollte doch erwarten, dass der Nn. erigens ebenso in der Cervix wie am Corpus uteri die Längsfasern motorisch und die Ringfasern hemmend beeinflusse. Ebenso wäre zu erwarten, dass die Nn. hypogastrici in gleicher Weise die Ringmuskeln der Cervix wie die des Uterus motorisch, und die Längsmusculatur beider Uterusabschnitte hemmend beeinflusse. Nun findet aber in der Cervix eine dem Corpus uteri entgegengesetzte Innervation statt. Den Grund hierfür glauben wir in dem Faserverlaufe der Musculatur dieser bejden Abschnitte des Uterus zu finden. 
Bekanntlich haben v. Hoffmann (12) und Bayer (11) vom genetischen Standpunkt aus den Verlauf der Muskelfasern des Uterus verfolgt und der complicirten Aufbau derselben erklärt. Ich kann hier nicht auf die Einzelheiten dieser Untersuchungen eingehen, will nur hervorheben, dass aus den Darstellungen dieser Autoren der Uebergang eines Theiles der Längsmusculatur des Uterus in die Ringmusculatur der Cervix hervorgeht.

So sagt H. Bayer (11): „dass den ganzen Gebärmutterhals entlang eine Anhäufung von Ringfasern sich vorfindet, in welcher er eine schleuderförmig angeordnetete Endausbreitung der Retractorenfasern der Muskelbündel der Ligamenta sacro-uterina erblickt." G. v. Hoffmann (12) nimmt an, "dass der Uterus aus der Verschmelzung der beiden Müller'schen Gänge entstehe, und dass seine Faserung deshalb zu den Muskelfaserlagen der Tuben in einer gewissen Verbindung stehe. Die äussere Längsfaserschicht der Tube breitet sich auf der Oberfläche des Uterus aus, und stellt die subseröse Muskelhaube des Fundus uteri dar. Die innere tubare Längsschicht bildet in ihrer Fortsetzung die spiralen Kreiszüge, welche die Tubenostien, den inneren Muttermund und den Cervicalcanal in Kreisen umziehen.

Auch Lott (13) hatte schon früher nachgewiesen, dass die selbstständigen Muskelbündel der Cervix aus dem Uteruskörper und der Vagina stammen. Die äussere Längsfaserschicht des Uterus scheint sich am inneren Muttermund zu theilen, indem ein Theil nach einwärts ziehend den inneren Muttermund schleuderartig umgiebt, und so die dort befindliche Ringmusculatur wesentlich verstärkt.

Der Vollständigkeit halber müssen wir auch die histologischen Untersuchungen der Cervix von Dührssen erwähnen. Nach diesem Forscher (14) zerfällt die Portio in zwei gleich grosse Abschnitte; einen, dem Cervicalcanal angrenzenden, von derberer Consistenz, mit reichlicher glatter Musculatur, aber fast ohne elastische Fasern, und einen grossen Abschnitt, der seine weiche Consistenz und sein starkes Retractionsvermögen seinem Reichthum an elastischen Fasern verdankt. Die Musculatur der Cervix besteht aus Ringund Längsfasern, erstere bilden die mittlere, letztere die innere und äussere Schicht.

Wie wir gesehen, wird ein Theil der Längsfasern des Uterus zu Ringfasern der Cervix, daher dieselben Innervationsimpulse, welche seitens des Erigens zu den Längsfasern des Uterus gleiten, ebenfalls auf die Ringfasern der Cervix übergehen und diese zur 
Contraction bringen, und ebenso die hemmenden Impulse, welche seitens desselben Nerven auf die Ringfasern des Uterus gleiten, auch auf die Längsfasern der Cervix einwirken und diese erschlaffen. Ein diesem entgegengesetztes Verhalten zeigt der N. hypogastricus.

Diese höchst auffallende Thatsache, dass die Wirkung beider Nerven auf das Corpus uteri und die Cervix, nicht, wie man erwarten sollte, in gleichem Sinne, sondern in antagonistischer Weise zutage tritt, erscheint nun im ersten Momente ganz unverständlich. Bei einiger Ueberlegung und bei Berücksichtigung der Vorgänge, welche am Uterus und in der Cervix in den einzelnen Phasen des Geburtsverlaufes und auch schon in den letzten Wochen der Gravidität, bei den sogenannten "Vorwehen" statt haben, wird dieser anscheinende Widerspruch aufgeklärt.

Es wird sich im Gegentheil zeigen, dass diese eigenthümlichen Innervationsverhältnisse sowohl während der Gravidität wie im Geburtsverlauf, ferner bei einigen physiologischen Vorgängen, z. B. bei der Cohabitation, eine wichtige Rolle spielen, wie ich dies noch auseinandersetzen werde.

Vorerst wollen wir noch einen Versuch am Uterus mit Aenderungen des Seitendruekes, und dann einen Versuch an der gespannten Vagina anführen.

\section{Versuch mit Aenderungen des Seitendrucks.}

Es wurde eine Canüle in die Cervix eingebunden und mit einem v. Basch'schen Wellenzeichner verbunden. Die Hörner wurden abgespert und es communicirte nur die Höhle des Uteruskörpers durch Wasser (warme Kochsalzlösung) mit dem Wellenschreiber. Der Uterus stand also unter positivem Drucke; sodass die Wand desselben i. e. sowohl die Längsfasern wie die Ringmuskelfasern durch den auf sie lastenden Flüssigkeitsdruck ausgedehnt wurden. Diese Versuche sind also vollständig analog jenen, in welchen der Lterus durch Gewichte belastet wurde.

Bei diesem Versuche waren am Uterus sehr deutliche spontane Bewegungen wahrnehmbar. Hier kam es nach starken elektrischen Strömen sowohl bei Hypogastricusreizung wie Erigensreizung nach vorausgehender Contraction, welche sich durch höhere spontane Contractionen kundgab, zu einer nachfolgenden Senkung und vollständigen Erschlaffung des Uterus. Die Feder zeichnete eine gerade Linie. Siehe Fig. 59 h, Hypogastricusreizung R. a. 6 25 Sek. und Fig. 60 i, Erigensreizung (R. a. 615 Sek.). 
Nur folgt bei Hypogastricusreizung sofort nach einer kurz dauernden Contraction eine Senkung unter den Ausgangspunkt und dann die Erschlaffung, während nach Erigensreizung vorerst eine langdauernde Contraction unter anfangs steigenden und später abnehmenden spontanen Contractionen auftritt, daher die Senkung allmälig erfolgt, und dann erst die langdauernde Erschlaffung eintritt — die Feder zeichnet eine gerade Linie unter den Ausgangspunkt.
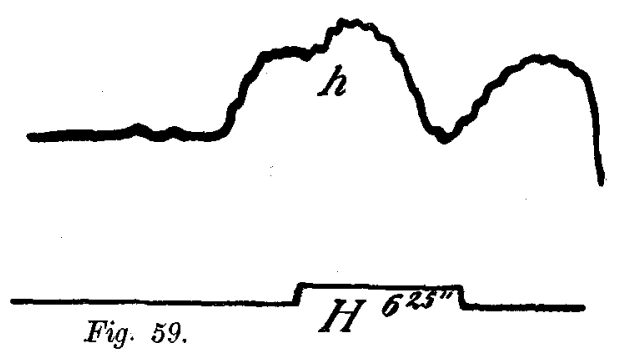

Ursprüngliche Grösse.

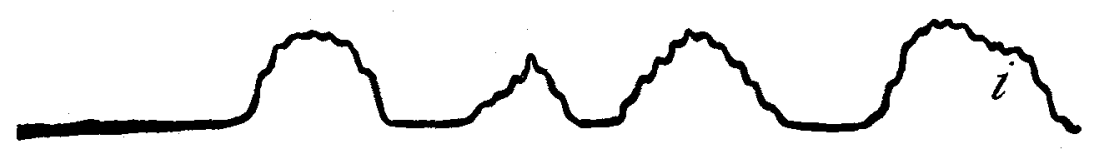

Fig 60 .

$\sqrt{E^{15}}$

Ursprüngliche Grösse.

Die Contraction nach Erigensreizung betrifft hier die Längsmusculatur und die nach Hypogastricusreizung die Ringmusculatur des Uterus.

Diese Erschlaffung nach der Reizung sowohl des einen wie des anderen Nerven weist auf eine Zunahme des Innenraumes des Uterus hin, in Folge des Nachlasses des Tonus beider Muskelfasersysteme, sowohl des der Längsmuskeln wie der Ringsmuskeln.

Zuweilen kommt es nach Hypogastricusreizung mit starken Strömen zu einer ganz ausgesprochenen langdauernden tetanischen Contraction unter kleinen Secundärwellen, so dass die Curvenlinie hoch ansteigt und unter rhythmischem Wellenspiel lange auf dem höheren Niveau verbleibt, und dann erst sich senkt (Fig. $61 \mathrm{f}$ R. a. 7, 20 Sek.). 
Hier kommt die Contraction der Ringmuskeln in markanter Weise zum Ausdruck, der Uterus ist in die Länge gestreckt, der Innenraum verengt. Ich werde bei meinen theoretischen Auseinandersetzungen auf diese Curve zurückkommen.

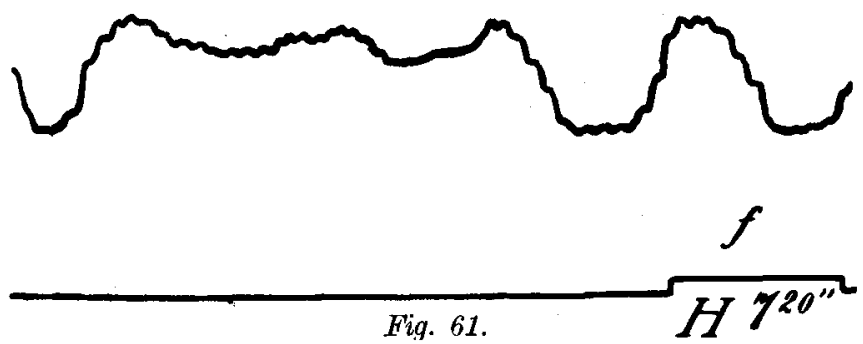

Ursprüngliche Grösse.

D. Versuch an der gespannten Vagina.

Es wurden zwei Nadeln senkrecht auf die Längsachse der Vagina eingestochen und mit der myographischen Zange verbunden, um die Bewegungen der Vaginalmusculatur bei Reizung des einen und des anderen Nerven zu studiren. Diese Versuche wurden an einem kleinen zarten Uterus vorgenommen.

a) Erigensreizung.

Nach Erigensreizung (Fig. 64 d, N. a. 6) kam es zuerst zu einem Wellenberg, dann zu einer langdauernden Senkung der Curve unter Secundärwellen. Der Wellenberg ist der Ausdruck der Contraction der Längsfasern der Vagina, die Senkung der Ausdruck des Nachlasses der Contraetion, aber auch weiterhin die Folge der hemmenden Wirkung des Erigens auf die Ringmuseulatur der Vagina, welche erst nach dem Nachlass der Contraction der Längsmuskeln in die Erscheinung tritt, wie wir ja das auch am Corpus uteri nachgewiesen haben. Die Secundärwellen zeigen, dass die Erschlaffung der Längsmuskeln von Strecke zu Strecke und nicht auf einmal sich vollzieht.

b) Hypogastricusreizung.

Nach Reizung der Hypogastrici (Fig. 65 g, R. a. 6) tritt nach geringer Contraction eine seichte Vertiefung ein, welcher wieder eine Contraction folgt. Die Contraction ist der Ausdruck der motorischen Wirkung der Hypogastrici auf die Ringmusculatur der 
Vagina und die seichte Senkung der Carvenlinie unter den Ausgang der Ausdruck der hemmenden Wirkung der Hypogastrici auf die Längsmusculatur der Vagina. Der Effect ist hier so gering, weil der Uterus

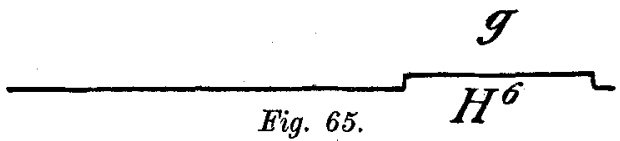

Ursprüngliche Grösse.

des jungfräulichen Thieres sehr zart und klein war. Auch Fig. 64, e, zeigt einen ähnlichen Effect.

Wir haben blos einen Versuch über die Innervation der Vagina aufzuweisen, doch lehrt schon dieser, dass die Innervation die gleiche ist wie beim Corpus uteri, nämlich:

1. Der $\mathbf{N}$. erigens ist der motorische Nerv für die Längsmusculatur der Vagina und der hemmende für die Ringmusculatur derselben.

2. Der N. hypogástricus ist der motorische Nerv für die Ringmusculatur der Vagina und der hemmende für die Längsmusculatur derselben.

\section{Theoretische Betrachtungen.}

Ich will nun auf Grundlage der aus unseren Untersuchungen hervorgegangenen Resultate die Art der Wirkungen der in Rede stehenden Nerven bei einigen physiologischen und pathologischen Vorgängen: dem Geburtsverlauf, der Cohabitation und der Atonie des nicht puerperalen Uterus, auseinanderzusetzen versuchen. 


\section{A. Geburtsvorgänge.}

An den Vorgängen des Geburtsaktes betheiligen sich sowohl beide Muskelfasersysteme des Corpus uteri wie auch jene der Cervix, jedoch tritt hierbei, wie unsere Untersuchungen lehren, ein doppelter Antagonismus zutage. Der eine Antagonismus zeigt sich in dem Verhalten des einen Muskelfasersystems gegenüber dem anderen desselben Uterusabschnittes, in dem Sinne, dass niemals beide Muskelfasersysteme gleichzeitig contrahirt bezw. gehemmt in die Erscheinung treten, sondern, dass das eine System erschlafft, wenn das andere sich contrahirt und vice versa. Der andere Antagonismus manifestirt sich in dem entgegengesetzten Verhalten der beiden Fasergattungen des Corpus gegenüber den Fasersystemen der Cervix. Aus unseren Untersuchungen geht ja hervor, dass einerseits der N. erigens gleichzeitig die Längsmusculatur des Corpus uteri und die Ringmusculatur der Cervix zur Contraction bringt und dabei die Ringmusculatur des Corpus und die Längsmusculatur der Cervix hemmt; und dass andererseits die Nn. hypogastrici gleichzeitig die Ringmusculatur des Corpus und die Längsmusculatur der Cervix motorisch beeinflussen und dabei die Längsmusculatur des Corpus und die Ringmusculatur der Cervix hemmen.

Nie tritt die Contraction resp. die Hemmung in beiden Fasersystemen desselben Uterusabschnittes gleichzeitig zutage, sondern abwechselnd. Das rhythmische Spiel von gegenseitig abwechselnder Contraction und Erschlaffung führt ja bei anderen, mit glatten Muskelfasern ausgestatteten, Hohlorganen z. B. beim Darme zur Peristaltik. Am menschlichen Uterus ist eine solche Peristaltik wegen des schnellen Ablaufes der einzelnen Wellen schwer nachweisbar.

Es haben jedoch ein Anzahl von Autoren eine peristaltische Bewegung wahrgenommen, so Heddäus (15), Spiegelberg Keiffer (16), Schatz (17) v. Winkel (18).

Kehrer (19) und Jastrebow (20) constatirten am Kaninchenuterus eine antiperistaltische Bewegung in Form ringförmig entstehender Zonen, welche vom Muttermunde zu den Eileitern und von da wieder zurückliefen. -- Auch wir haben bei Hündinnen sowohl peristaltische wie antiperistaltische Bewegungen an beiden Hörnern und an der Vagina in ähnlicher Weise beobachtet.

Werth (21) fühlte, wenn er die Hand gleich nach Aus- 
stossung der Frucht in den Uterus einführte, wie der unterste Theil sich zuerst zusammenzog, während der Fundus noch schlaff war und dass die Wehencontraction dann aufwärts stieg, während die untere Partie erschlaffte.

Ein gleichzeitig allseitiges Auftreten der Contraction beim Menschen nehmen folgende Autoren an: Ahlfeld (22), Cohnstein (23) Gläwecke, v. Hoffmann (24), Werth, Schäffer (25).

lch glaube aus unseren Untersuchungen Belege für die verschiedenen Anschauungen erbringen zu können, sowohl für das Auftreten einer allseitigen Contraction, wie einer peristaltischen und auch einer antiperistaltischen Contraction.

Wenn die Wirkung des Erigens vorherrscht, kommt es zur peristaltischen Contraction, wenn die Wirkung der Hypogastrici prävalirt, kommt es zur antiperistaltisehen Contraction, und wenn beide Nerven abwechselnd und gleichmässig wirken, kommt es zum allseitigen Auftreten der Contraction.

Wie die Vorgänge sich hierbei abspielen, und ob diese verschiedenen Arten der Contraction während des ganzen Geburtsverlaufes oder nur in den einzelnen Perioden desselben gesondert in die Erscheinung treten, werde ich später auseinandersetzen. Vorerst will ich in Erinnerung bringen, wo die peripheren Nervencentren sind, von welchen auf dem Wege des Reflexes die Impulse der abgehandelten Nerven ausgelöst werden. Das sind bekanntlich die zahlreichen Ganglien, welche im Fundus und Corpus uteri in der Nähe der Tubeninsertion sich befinden, ferner das Ganglion cervicale über dem hinteren Scheidengewölbe, an beiden Seiten des Collums, endlich die paracervicalen Ganglien Knüppfer's, welche in der Höhe der Scheideninsertion sich befinden.

Die Ganglien im Fundus und Corpus uteri stehen mit den Sacralnerven, welchen der N. erigens entstammt, in Verbindung, während die Cervicalganglien, unter anderen Nerven, die Nn. hypogastr. enthalten.

Der Effekt beider Nerven ist nicht nur in muskulo-motorischer Beziehung - die Locomotion des Uterus und der Cervix und die Formveränderung des Uterus betreffend -, sondern auch in vasomotorischer Beziehung verschieden.

Wenn der Erigens wirkt, contrahirt sich der Uterus nach unten, weil die Längsmuskulatur sich zusammenzieht, gleichzeitig erschlafft aber die Ringmuskulatur des Uterus, infolge hiervon erweitert sich der Uterus in der Querrichtung. Es ist ja bekannt, 
dass bei den Wehen der sagittale Durchmesser zunimmt. Der Inhalt des Uterus wird diesem Drucke ausweichen. Frucht und Fruchtwasser werden sich verschieben. Gleichzeitig contrahirt sich die Ringmuskulatur der Cervix, während die Längsmuskulatur derselben sich verlängert. Dieses letztere Phänomen ist ja von einigen Autoren während der Wehen festgestellt worden: Breisky. (26), Schäffer (27). Beide Nerven haben aber neben den muskulo-motorischen Wirkungen, wie wir oben sagten, auch eine vasomotorische. Der Erigens führt Vasodilatatoren und der Hypogastricus Vasoconstrictoren (v. Basch und Hoffmann). Bei der Erigensreizung wird daher die Blutzufuhr zum Uterus und der Cervix erhöht. Diese führt zur Auflockerung und Schwellung der Schleimhaut und Erschlaffung der Gewebe der Cervix. 0. Fellner-Schauta (28), Schröder (29), und Wiesner (30) haben eine Erhöhung des Blutdruckes schon während der letzten Monate der Schwangerschaft festgestellt, welche sich während der ganzen Geburt weiter steigert.

Die Frage ist nun: Ja kommt es denn während der Wehen überhaupt vor, dass der Uterus sich nach unten contrahirt und tiefer in das Becken hinabsteigt? Einzelne Autoren beschreiben diese Art des Vorganges, Schäffer, und v. Hoffmann sagt in. seinen morphologischen Studien über die. Wirkung der äusseren Faserschichte während der Geburt: „Da die Blätter von innen unten nach aussen oben aufsteigen, so muss sich ihre Contractionswirkung gegen den inneren Muttermund concentriren. Denkt man sich ausserdem noch, dass die Blätter sich gegeneinander verschieben, so wird bei ihrer Contraktion der Uterus seine Form dahin abändern, dass der Fundus tiefer herabgedrückt wird. Die äussere Schichte schiebt sich etwa wie ein Fernrohr in sich selbst zusammen.".

Ueberdies weist ja die von den meisten Autoren hervorgehobene Tiefenzunahme des Uterus während der Wehe auf Erigenswirkung hin, denn die Hypogastrici wirken ganz anders, wie wir gleich beschreiben werden.

Wenn die Hypogastricuswirkung überwiegt, verkürzen sich die Ringmuskeln des Uterus, sein Querlumen wird verengt. Da aber gleichzeitig die Längsmuskeln des Uterus erschlaffen, verlängert sich das Organ in verticaler Richtung, der Fundus tritt hoch binauf, der Uterus streckt sich und bäumt sich auf, hierdurch. wird auch das Kind gestreckt, was ja allgemein bekannt ist. Da aber 
der Hypogastricus auch hemmende Nerven für die Ringmusculatur der Cervix führt, wird der Tonus dieser Musculatur herabgesetzt, und da mit der Streckung des Uterus das untere Uterinsegment und auch die Cervix in die Höhe gezogen werden, muss mit jeder neuen Wehe die Cervix immer mehr entfaltet werden. Der untere Eipol wird in Folge der Zunahme des Innendruckes in den Locus minoris resistentiae - die Cervix - immer tiefer hineingepresst. Hierzu kommt, dass mit der Hypogastricuswirkung auch eine Contraction der Längsmuskeln der Cervix eintritt - denn der Нypogastricus ist ja der motorische Nerv dieser Musculatur - die Vaginalportion wird daher auch mit jeder durch Hypogastricuswirkung ausgelösten Wehe immer mehr verkürzt.

Meine experimentellen Untersuchungen über den Seitendruck stellen ja eine Analogie zu den Vorgängen dar, welche bei der Geburt statthaben, da sie zeigen, dass sowohl nach Erigens- wie nach Hypogastricusreizung der Innendruck steigt, um nach der Contraction abzusinken. Bei der complicirten Structur der Uterusmusculatur, an welcher Längsfasern, Ringfasern und schief aufsteigende Fasern sich vielfach kreuzen, ist wohl anzunehmen, dass die Contraction der Ausdruck mehrerer Componenten ist und die Resultante sowohl der hemmenden wie der motorischen Nerveneinflüsse darstellt.

Besonders lehrreich in Bezug auf die Hypogastricuswirkung ist Fig. $61 \mathrm{f}$, welche eine Curve darstellt, in welcher es zu einer langdauernden hohen Contraction der Ringmusculatur kommt, welche nahezu tetanischen Charakter zeigt und eine Analogie zu den starken Wehen, welche gegen Ende der Eröffnungsperiode auftreten, bildet.

Die Frage, ob während des ganzen Geburtsverlaufes beide Nerven abwechselnd in Action treten, oder $o b$ in den einzelnen Phasen bloss die Wirkung eines der beiden Nerven vorwiegt, vermag ich nicht strikte zu beantworten, da ich dies weder aus den Wehencurven, welche von Schatz (31), Westermark (32), Schäffer u. A. aufgenommen wurden, noch aus den meinigen zu ersehen vermag. Allerdings habe ich eine Curve aufzuweisen, in welcher mitten in die Erigenswirkung die Hypogastricusreizung einfiel und das Resultat der gleichzeitigen Wirkung beider Nerven demonstrirt, daher die allseitig wirkende Uteruscontraction illustrirt. Es ist dies Fig. $36 \mathrm{c} \mathrm{d}$. Hier wurde gleichzeitig der Effect beider Nerven sowohl auf das Corpus uteri als auch auf die Cervix aufgenommen, jedoch derart, dass die Hypogastricusreizung erst nach 
einem längeren Intervall der Erigensreizung folgte. Da sehen wir im Corpus uteri eine sehr breitgipflige, wellenförmig verlaufende Contraction, welche die Resultante der Componenten beider Nervenreizungen ịst. Sie ist minder hoch als nach Reizung des Nervus erigens allein, ist jedoch von grosser Erschlaffung gefolgt. Und in der Cervix sehen wir einen ähnlichen Effect, nur der geringeren Muskelmasse entsprechend, weniger ausgeprägt. Doch möchte ich dieses Ergebniss nicht ohne weiteres auf die Geburtswehen übertragen, es sollte nur ein annäherndes Bild sein, es entspricht ja nicht ganz den Verhältnissen bei der Geburt, denn es fehlte der Uterusinhalt, die Frucht, so dass es nicht zu Druck und Gegendruck kam.

Der dargestellte Effect in der Cervix entspräche eher noch jenem Vorgänge, bei welchem die austretende Frucht in die Vagina gelangt und nur das untere Ende derselben sich noch in der Cervix befindet, denn auch hier war ein fremder Körper, die Zange, in der Cervix.

Mit aller Reserve möchte ich jedoch der Vermuthung Ausdruck leihen, dass bei den „Dolores praesagientes" die Wirkung der Erigentes vorwaltet, da hierbei ein Herabrücken des Uterus eintritt und die oben erwähnte vasodilatatorische Wirkung zur Geltung kommt, welche zur Schwellung und serösen Durchfeuchtung des Cervixgewebes führt.

Bekanntlich implantirt sich das Ovulum in den meisten Fällen im Fundus und in der Nähe der Tubenmündungen. In der ersten Hälfte der Schwangerschaft findet hauptsächlich ein actives Wachsthum des Uterus statt, während in der zweiten Hälfte der Uterus durch die Wachsthumszunahme des Eies gedehnt wird. Diese Dehnung erstreckt sich auch auf den Fundus uteri und übt einen Reiz auf die im Fundus und Corpus befindlichen subserösen Ganglien aus. Von diesen Ganglien wird der Reiz auf die Nervi erigentes übertragen und es kommt anfänglich zu schwachen, seltenen Contractionen des Uterus, allmählich aber zu stärkeren, den sogenannten "Vorwehen".

In der zweiten Hälfte der Schwangerschaft wächst das Ei immer mehr gegen die Cervix zu und der untere Eipol gelangt allmählich in die Gegend der Ganglien, welche sich über dem Scheidengewölbe befinden. Indem nun die durch die Erigenswirkung hervorgerufenen Contractionen den unteren Eipol an diese Ganglien and̆rücken, werden von diesen aus die Nervi hypo- 
gastrici in Action gesetzt, welche, wir wir später auseinandersetzen werden, die eigentlichen Geburtswehen auslösen.

Als Beweis dessen, dass rom Fundus uteri Contractionen ausgelöst werden, sprechen die Wehen bei Graviditas extrauterina und bei Abortus in jenen Fällen, wo das $\mathrm{Ei}$ nahe dem Fundus sass, wie der von Fischel (33) erwähnte Fall, in welchem das Abortivei schon wenige Tage nach dem Ausbleiben der Menstruation ausgestossen wurde. "Das von der Circumflexa bekleidete Ei hatte nach oben im rechten Horn gelegen" (Schäffer l. c.)

Was das Herabrücken des Uterus hetrifft, sagt Schäffer „Eine Serie verstärkter Schwangerschaftswehen, der Vorwehen, oder, zumal bei Mehrgebärenden, die ersten Geburtswehen bringen das Tiefertreten des ganzen Uterus in das Becken hinein zustande".

In der Eröffnungsperiode zeigt sich sehr deutlich die Wirkung der Hypogastrici wie uns unsere Fig. 27 illustrirt. Die Ringmuskulatur des Corpus uteri contrahirt sich, der Uterus wird gestreckt, er bäumt sich auf, das untere Uterinsegment und die Cervix werden nach oben gezogen und da gleichzeitig die hemmende Wirkung der Hypogastrici die Ringsmusculatur der Cervix zur Erschlaffung bringt, erweitert sich der innere Muttermund und die Cervix wird allmählich entfaltet. Doch tritt dabei auch die Wirkung der Erigentes zutage, denn der Fundus folgt stets dem herabrückenden Kinde, liegt dem Steisse immer an. „Erst beim Durchschneiden des Kopfes durch die. Vulva wird die Berührung zwischen Steiss und Fundus uteri aufgegeben. Der Hohlmuskel zieht sich nach der Bauchseite der Frucht zurück und dabei werden auch die Beine des Kindes zurückgestreift und gestreckt" sagt E. Bumm (34).

Nach Litzmann (35), Schröder (36) Schatz, Werth (37) u. A. soll aber ein Höhersteigen des Fundus eintreten, welches durch die Rückzugsbewegung des Uterus selbst bewirkt wird. Dies weist auf Hypogastricuswirkung hin. Auch die Anschauung Schäffer's, dass vor dem Blasensprunge das $\mathrm{Ei}$ in toto und nach dem Blasensprunge, die Frucht in die Länge gestreckt wird, kann durch die Hypogastricuswirkung erklärt werden, weil die Contraction der Ringmusculatur des Corpus uteri eine Höhenzunahme des Uteruslumens und eine Streckung des Eis, bezw. der Frucht bewirkt.

In der Austreibungsperiode, in welcher die Bauchpresse mitwirkt, scheint ebenfalls die Hypogastricuswirkung vorzuherrschen, 
264 Fellner, Die Bewegungs- und Hemmungsnerven des Uterus.

weil auch hier der Fundus höher hinaufsteigt, sodass nach Litzmann (35) und Schröder (36) der Gebärmuttergrund zuletzt höher als der Rumpf des Kindes steht. Dem widerspricht jedoch v. Winckel (38), einerseits auf Grund seiner ejgenen Beobachtungen, nach welchen der Fundus unmittelbar nach der Geburt des Kindes, nicht wie behauptet wird, 3-4 Finger breit oberbalb des Nabels, sondern in Nabelhöhe steht, andererseits auf Grund der Gefrierschnitte von Braune, (39) Barbour und Webster (40), Chiari (41), Zweifel (42), u. A., welche die Verhältnisse der Austreibungsperiode darstellen. Dass auch Ernst Bumm dem widerspricht, habe ich oben angeführt. Es würde sich demnach auch der $\mathrm{N}$. erigens in der Austreibungsperiode aktiv mit betheiligen müssen, wenn auch nicht in dem Masse wie der Hypogastricus. In der Nachgeburtsperiode wirken sicherlich beide Nerven abwechselnd zusammen, denn der Uterus contrahirt sich unter normalen Umständen allseitig, auch nach unten, und zwar scheint hier wieder der Erigens zu prävaliren.

\section{B. Die Atonie des nicht puerperalen Uterus.}

Auch die Ursache der in der letzten Zeit vielfach discutirten nAtonie des nicht puerperalen Uterus" [Kossmann (43), Tussenbroek (44), Schäffer (45), Asch (46) u. A.] lässt sich durch die Wirkung der beiden Nerven erklären.

Bekanntlich unterscheidet Schäffer zwei Grade: das Stadium der ballonförmigen Auftreibung mit vermindertem, aber nicht gänzlich verlorenem Tonus, und das Stadium der vollkommenen Erschlaffung, der Paralyse. Zu dieser soll es kommen, wenn pathologische Veränderungen am Uterus oder seinen Adnexen vorhanden sind, oder der Allgemeinzustand der Patienten zum Eintritt der Atonie disponirt.

Nach meiner Anschauung, welche ich in einer anderen Publication ausführlich begründen will ${ }^{1}$ ), wird das erste Stadium der Atonie durch die Dilatation der Cervix herbeigeführt, und das zweite Stadium durch die Excochleation, beide Stadien jedoch bei gleichzeitigem Bestehen der von Schäffer angegebenen disponirenden Momente.

Durch die Dilatation werden die Cervicalganglien gedrückt, hierdurch die Nn. hypogastrici vorübergehend gelähmt, der Tonus

1) Centralblatt f. Gynäkologie. 1906. No. 26. 
der Ringmusculatur des Corpus uteri wird aufgehoben, die Uterushöhle wird im Querdurchmesser erweitert. Da aber der Erigens intact bleibt, behält die Längsmusculatur des Corpus ihren normalen Tonus, daher erscheinen die Uteruswände verdickt. Hierzu kommt, dass auch die vasoconstrictorische Wirkung der Nn. hypogastrici gehemmt wird, während die vasodilatatorische Wirkung des Erigens fortbesteht, in Folge hiervon kommt es zu einem bedeutenden Afflux zum Uterus, welcher zur Schwellung und Verdickung der Uteruswände führt.

Dass die Uterusgefässe einen Antheil an diesen Vorgängen haben, hat ja auch Schäffer angenommen, und Frl. von Tussenbroek hat den Einfluss derselben- allein zur Erklärung dieser Vorgänge herangezogen.

Wenn nun das Curettement folgt, werden die Ganglien im Fundus und Corpus uteri in intensiver Weise gerejzt, es tritt Lähmung der Nervi erigentes ein, und nun verliert auch die Längsmusculatur des Uterus ihren Tonus, der Uterus erweitert sich auch in verticaler Richtung, es kommt zur allgemeinen Atonie, oder wie einige Autoren sagen: zur Paralyse.

C.

Ich möchte aber für das 1 . Stadium die Bezeichnung ${ }_{n}$ Hypotonie" und für das Il. Stadium die Benennung "Atonie" vorschlagen, da es sich doch nur um vorübergehenden Verlust des Tonus der Uterusmusculatur handelt.

Dass die Narkose ebenfalls ein disponirendes Moment hierzu

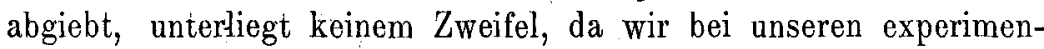
tellen Studien gesehen, dass die Wirkung der Uterusnerven versagte, wenn wir zu stark curaresirt hatten, oder in jenen Versuchen, in welchen wir starke Morphiuminjectionen vorgenommen hatten.

Ich muss hier noch die wichtige functionelle Bedeutung aufklären, welche jenem anatomischen und physiologischen Räthsel zu Grunde liegt, welches wir bei der Innervation der Cervix kennen gelernt, nämlich, dass sich die motorischen und hemmenden Nerveneinflüsse auf die Cervixmusculatur entgegengesetzt $\mathrm{zu}$ jenen verhalten, welche bei der Musculatur des Corpus uteri sich manifestiren.

Diese anatomische und physiologische Einrichtung hat nach meiner Neinung unter Anderem die wichtige Aufgabe, eine ungeeignete Eiimplantation $\mathrm{zu}$ verhindern und die Frucht vor $\mathrm{zu}$ frühem Austritt zu schützen. 
Der Sphincter internus bildet eine Barrière zwischen Uterus und Cervix, welche verhütet, dass das befruchtete $\mathrm{Ei}$ sich an einer Stelle einniste, welche für seine volle Entwickelung zur reifen Frucht ungeeignet ist, z. B. die Cervix. Das beweisen die seltenen Fälle von Placentabildung in der Cervix, während die Placenta praevia centralis, partialis und lateralis doch relativ häufiger vorkommen.

Auch bei den "Dolores praesagientes", welche nach meiner Meinung ausschliesslich vom Erigens ausgeher, wie ich das ja auseinandergesetzt habe, wird mit jeder Contraction der Längsmuskeln des Uterus sich der Sphincter gleichzeitig contrahiren und die Barrière wird verstärkt. Erst in der Eröffnungsperiode tritt der Nervus hypogastricus in Action, wie wir oben auseinandergesetzt.

Setzen wir nun den Fall, der Faserverlauf der Musculatur wäre im Corpus uteri und in der Cervix gleichartig, sodass jener Theil der Längsmusculatur des Corpus, welcher zur Ringmusculatur der Cervix wird, ebenfalls Längsmusculatur bliebe, was würde da geschehen? Bei jeder Erigenswirkung würde die Längsmusculatur beider Uterusabschnitte in Contraction gerathen und das Ei nach abwärts drängen und der untere Eipol geriethe frühzeitig in die Gegend des Scheidenansatzes und drückte auf die daselbst befindlichen Ganglien und, indem nun auch die Hypogastrici in Action kämen, müsste es in allen Fällen von Gravidität schon frühzeitig zur Ausstossung der Frucht kommen.

\section{Cohabitation.}

Welche Aufgabe erfüllt nun diese Barrière bei der Cohabitation und wie macht sich der Einfluss beider Nerven bei diesem Vorgange geltend?

Die Friction an der Clitoris, dem "Wollustorgane", und im Introitus vaginae lösen mechanisch, und die erotischen Gefühle psychisch, jene Nervenimpulse aus, welche bei der Cohabitation zu motorischen und vasomotorischen Vorgängen führen. Dabei scheint wieder die Wirkung des Erigens, wie schon sein Name andeutet, in der ersten Phase des Aktes vorzuherrschen. Vermöge seiner vasodilatatorischen Wirkung, wie dies von v. Basch und Hoffmann zuerst nachgewiesen wurde, werden die Gefässe des Uterus sowohl, wie der Schwellkörper der Clitoris strotzend mit arteriellem Blute gefüllt. Gleichzeitig werden die motorischen Fasern des Erigens die Längsmusculatur des Corpus zur Contrac- 
tion bringen, und die hemmenden Fasern die Ringsmusculatur des Uterus erschlaffen, so dass die Lichtung der Uterushöhle im Längsdurchmesser zwar abnimmt, aber der Quere nach zunimmt. Durch die Hyperaemie des Corpus und der Cervix wird das Gewebe der letzteren succulent und für die später eintretende motorische Action der Hypogastrici, welcher zur Eröffnung des Sphincters, der Beseitigung der Barriere führt, vorbereitet. Während der ersten Phase der Cohabitation scheint von Seiten der No. hypogastrici nur die vasoconstrictorische Wirkung sich geltend zu machen, wodurch die Abfuhr des venösen Blutes beschränkt wird. Wenn nun der Orgasmus den Höhepunkt erreicht hat, ist der Erigens erschöpft, die Contraction der Längsmusculatur des Corpus sowohl, sowie die der Ringsmusculatur der Cervix lässt nach, und gleichzeitig setzen die motorischen Impulse der $\mathrm{Nn}$. hypogastr. ein, die Ringmusculatur des Corpus contrahirt sich, der Uterus streckt sich, und die hemmenden Fasern der Hypogastrici bringen die Ringmusculatur der Cervix zur Erschlaffung. Die Barière öffnet sich, Ostium int. und extern. klaffen, der Cervicalcanal bildet eine offene Höhlung, und der Weg für das Eindringen des Spermas in die Cervix wird frei.

Die Auslösung der Hypogastricus-Impulse wird möglicherweise durch den Reiz, welchen die Friction des Penis auf die in dem oberen Theile der hinteren Vaginalwand befindlichen Ganglien ausübt, herbeigeführt.

Wenn nun der Orgasmus bei Mann und Weib synchronisch eintritt, wird das Sperma in die Cervix injaculirt, und in die Uterushöhle aspirirt, weil ihr Lumen durch die hemmenden Fasern der Hypogastrici auf die Längsmusculatur in verticaler Richtung erweitert wird.

Tritt der Orgasmus bei Mann und Weib nicht gleichzeitig ein, so wird das Sperma in der Vaginalbucht verbleiben und die Zoospermen gelangen durch Eigenbewegung in die Cervix und nachher in die Uterushöhle. Die deletäre Wirkung des sauren Vaginalsecretes wird durch den Schleim neutralisirt, welcher infolge der Hyperaemie in vermehrter Menge von den Schleimdrüsen der Uterusmucosa abgesondert wurde und bei der Contraction der Ringmusculatur des Corpus zu Beginn der Action der Hypogastrici herausgeschleudert wurde - die sogenannte Ejaculation beim Weibe!

Die selbstständige Locomotion der Spermatozoen wird bekanntlich darum angenommen, weil die Flimmerbewegung der 
Epithelien im Uterus und in den Tuben [nach Hofmeier (47) und L. Mandl (48) u. A.] nicht nach aufwärts gegen die Tuben gerichtet ist, sondern nach abwärts und daher dem Eindringen der Zoospermien eher hinderlich wäre. Nach Seligmann wird auch eine chemotactische Wirkung angenommen.

Man hat die Möglichkeit der directen Injection des Spermas in die Uterushöble bei der Ejaculation aus mehreren Gründen bestritten. Erstens weil die Achse des Uterus mit jener- des Penis ein Zusammentreffen des Ost. extern. mit der Mündung der Harnröhre nicht möglich mache. Zweitens weil der Cervicalcanal nicht klafft und kein leerer Raum in der Höhle des Cervicalcanals besteht, besonders bei Virgines, wo die Cervixwände fest aneinanderliegen. Drittens wären die Fälle, dass bei Virgines, ohne dass der Penis in die Vagina eindringt, und trotz des unversehrt bestehenden Hymens eine Coception stattfinde, nicht denkbar.

Dem kann man nun entgegenhalten, dass bei der Cohabitation der Uterus und die Cervix ihre Gestalt und Lage verändern, ersterer sich dem Penis nähert und seine Achse sich dreht, weil die Cervix sich verlängert und nach vorne wendet, wie die Untersuchungen von v. Basch und Hoffmann im Thierexperimente nachgewiesen. Das Orif. extern. klafft und auch Cervicalcanal und Orif. intern. werden im 2. Stadium in Folge der Hypogastricuswirkung durch die hemmende Wirkung der Hypogastrici auf die Ringmusculatur der Cervix erweitert, so dass selbst bei Virgines, wenn nicht Anomalien oder pathologische Veränderungen vorliegen, sich eine Höhle bilden kann, weit genug, um dem Sperma den Eintritt zu gestatten. Hierdurch werden die Punkte 1 und 2 erklärt. Was den Punkt 3 betrifft, so dürften in diesen seltenen Fällen die Cervixwände vielleicht von vornherein nicht so fest aneinanderliegend gewesen sein, um den Spermatozoen, welche vermöge ihrer Eigenbewegung durch eine Lücke des Hymens eindringen konnten, die Passage in den Cervicalcanal und die Uterushöhle zu sperren. Die Erfolge der Erweiterung der Cervix bei der Sterilität sprechen doch für die directe Injection des Spermas in die Cervix (Chrobak und v. Rosthorn) oder die Möglichkeit des nachträglichen Eindringens.

Bei Frauen, die geboren haben, ist ja der Cervicalcanal ohnehin erweitert, und das Ostium externum bildet einen offenen Querspalt.

Nachdem die Cohabitation zu Ende gegangen, erschlafft auch die Ringmusculatur des Uterus und die normalen Innervationsim- 
pulse beider Nerven treten wieder ein, so dass der normale Tonus beider Muskelfasersysteme sich allmälig wieder herstellt. Der Sphincter internus schliesst sich wieder und wird nachträglich verhindern, dass das ausgetretene Orulum, welches auf seinem Wege befruchtet wird, sich an einer Stelle einniste, wo die Bedingung für seine Entwicklung ungünstig sind, wie in der Cervix.

\section{Lit eratur.}

1. v. Basch u. Hoffmann, Wiener med. Jahrbücher d. k. k. Gesellschaft der Aerzte.

2. Leopold Fellner, Ueber die Bewegungs- und Hemmungsnerven des Uterus. Centralbl. f. d. med. Wissenschaften. 1887.

3. Kurdinowski, Physiologische und pharmak. Versuche an der isolirten Gebärmutter. Dieses Arch. 1904. Bd. 73. H. 2.

4. Spiegelberg, Zeitschr. f. d. rationelle Med. 1858.

5. Frankenhäuser, Nerven der Gebärmutter. Jena. 1867.

6. Kehrer, Ueber die Zusammenziehungen des weiblichen Genitalcanales. Habilitationsschrift. Giessen. 1863.

7. Körner, Studien des physiol. Instituts Breslau. 1865.

8. Leopold Fellner, Med. Jahrb. d. k. k. Gesellsch. d. Aerzte. Wien. 1884.

9. Derselbe, Arch. f. d. ges. Physiologie. Bd. 56. 1894.

10. Oser, Die lnnervation d. Pylorus. Jahrb. d. k. k. Gesellsch. d. Aerzte. Wien. 1884.

11. Bayer, Zur physiol. und morphol. Pathologie der Gebärmutter. Gynäk. Klinik. Strassburg. 1885.

12. G. v. Hoffmann, Morphol. Untersuchungen über die Musculatur des Uteruskörpers. Zeitschr. f. Geburtsh. u. Franenkrankh. 1876.

13. G. Lott, Anatomisches und allgemein Physiologisches über die Cervix uteri. Erlangen. 1872.

14. Dührssen Dieses Archiv. Bd. 41.

15. Heddäus, Contraction der Gebärmutter in physiol. Beziehung. Dissert. Würzburg. 1851.

16. Ke iffer, In Frommel's Jahrb. 1899.

17. Schatz, Geburtsmechanik bei Kopfendlagen. Leipzig. 1868.

18. v. Winckel, Lehrbach der Geburtshülfe.

19. Kehrer, Beiträge 1 u. 2.

20. Jastreboff, Arch. f. Anat. u. Phys. (Phys. Abth.) 1884.

21. Werth, In Müller's Handbuch d. Geburtsh. I. S. 335.

22. Ahlfeld, Wehensteigerung durch die Bauchpresse. Dieses Arch. V.

23. Cohnstein, Dieses Arch. 18.

24. v. Hoffman n, Zeitschr. f. Geb. u. Gyn. I. 472.

25. Schaeffer, Im Handb. d. Geburtsh. von v. Winckel. I. Bd. 2. Hälfte. 1904. 
26. Breisky, Krankheiten der Vagina. D. Chirurgie. Stuttgart. 1886.

27. Schaeffer, Im Handb. d. Geburtsh. von v. Winckel. I. Bd. 2. Hälfte. 1904.

28. 0, Fellner, Monatsschr. f. Geb. u. Gynäk. 1902. 5.

29. H. Schröder, Verh. d. gyn. Congresses. Giessen. 1901.

30. Wiesner, Gesellsch. f. Geburtsh. Leipzig. 1899. 470.

31. Schatz, Experimente über d. Wehentbätigkeit. Berlin. 1896. (Hirschwald.)

32. Westermark, Skand. Arch. f. Physiol. 1892. IV.

33. Fischel, Prager med. Wochenschrift. 1898. 48.

34. Ernst Bumm, Grundriss zum Studium der Geburtshülf $\theta$. III. Aufl.

35. Litzmann, Dieses Archiv. 12.

36. K. Schröder, Schwangerschaft, Geburt und Wochenbett. Bonn, 1867.

37. Werth u. Cobr, Müller's Handbuch 1.

38. v. Winckel, Monatsschr. f. Geburtsh. 20, 21.

39. v. Braune, Lage des Uterus und Fötus am Ende der Grav. Leipzig 1872.

40. Barbour u. Webster, Sect. anat. of labor. Edinburgb. med. journ. 1887. 1896.

41. Chiari, Ueber das topogr. Verhalten d. Genital. einer intra partum verstorbenen Primipara. Wien. 1885.

42. Zweifel, Zwei neue Gefrierschnitte Gebärender. Leipzig. 1893.

43. Kossman n, Centralbl. f. Gyn. 1904. 44 und 1905. No. 41.

44. Tussenbroeck, Centralbl. f. Gyn. 1905. 39 und 1906. No. 2.

45. Schäffer, Centralbl. f. Gyn. 1905. No. 40.

46. Asch, Centralbl. f. Gyn. 1905. No. 41.

47. Hofmeier, Zur Kenntniss der normalen Uterusschleimhant. Centralbl. f. f. Gyn. 1893. 33.

48. L. Mandl, Ueber die Richtung der Flimmerbewegung im menschlichen Uterus. Centralbl. f. Gyn. 1898. No. 13. 